\title{
The Clinical Utilisation of Respiratory Elastance Software (CURE Soft): a bedside software for real-time respiratory mechanics monitoring and mechanical ventilation management
}

\author{
Akos Szlavecz ${ }^{1 *}$, Yeong Shiong Chiew ${ }^{2 *}$, Daniel Redmond ${ }^{2}$, Alex Beatson ${ }^{2}$, Daniel Glassenbury ${ }^{2}$, Simon Corbett ${ }^{2}$,
} Vincent Major ${ }^{2}$, Christopher Pretty ${ }^{2}$, Geoffrey M Shaw ${ }^{3}$, Balazs Benyo ${ }^{1}$, Thomas Desaive ${ }^{4}$ and J Geoffrey Chase ${ }^{2}$

\author{
* Correspondence: \\ szlavecz@gmail.com \\ yeongshiong.chiew@canterbury.ac.nz; \\ 'Department of Control \\ Engineering and Information, \\ Budapest University of Technology \\ and Economics, Budapest, Hungary \\ ${ }^{2}$ Centre for BioEngineering, \\ University of Canterbury, \\ Canterbury, New Zealand \\ Full list of author information is \\ available at the end of the article
}

\begin{abstract}
Background: Real-time patient respiratory mechanics estimation can be used to guide mechanical ventilation settings, particularly, positive end-expiratory pressure (PEEP). This work presents a software, Clinical Utilisation of Respiratory Elastance (CURE Soft), using a time-varying respiratory elastance model to offer this ability to aid in mechanical ventilation treatment.

Implementation: CURE Soft is a desktop application developed in JAVA. It has two modes of operation, 1) Online real-time monitoring decision support and, 2) Offline for user education purposes, auditing, or reviewing patient care. The CURE Soft has been tested in mechanically ventilated patients with respiratory failure. The clinical protocol, software testing and use of the data were approved by the New Zealand Southern Regional Ethics Committee.

Results and discussion: Using CURE Soft, patient's respiratory mechanics response to treatment and clinical protocol were monitored. Results showed that the patient's respiratory elastance (Stiffness) changed with the use of muscle relaxants, and responded differently to ventilator settings. This information can be used to guide mechanical ventilation therapy and titrate optimal ventilator PEEP.

Conclusion: CURE Soft enables real-time calculation of model-based respiratory mechanics for mechanically ventilated patients. Results showed that the system is able to provide detailed, previously unavailable information on patient-specific respiratory mechanics and response to therapy in real-time. The additional insight available to clinicians provides the potential for improved decision-making, and thus improved patient care and outcomes.
\end{abstract}

Keywords: Mechanical Ventilation, Software, Respiratory Mechanics, Monitoring, Decision Making, Positive End-Expiratory Pressure (PEEP) 


\section{Background}

Patients with respiratory failure require mechanical ventilation (MV) for breathing support. In particular, patients with acute respiratory distress syndrome (ARDS) are mechanically ventilated to maintain alveoli recruitment allowing better oxygenation, reducing their work of breathing and aiding recovery. The causes of respiratory failure are often different between patients, and more importantly, responses to treatment can be highly patient-specific. Thus, there is a patient-specific optimal ventilator setting that, if determined, could potentially improve patient care and outcome [1,2].

MV supports patients' work of breathing until the underlying disease is resolved. However, there is little or no consensus to select patient-specific optimal ventilator settings with several iterations of the ARDSNet tables that several, but not all, clinicians use as a basis $[3,4]$. Clinicians often resort to experience, intuition or generalised approaches to select MV settings [5-9]. Thus, a great deal of research seeks to aid clinicians in optimising patient-specific ventilator setting.

In one case, a model-based approach is being investigated to select best positive end expiratory pressure (PEEP) [2]. PEEP is the additional pressure applied at the end of expiration to recruit and retain collapsed alveoli at expiration [10]. Studies have revealed that higher PEEP can be beneficial for ARDS patients [6], but high PEEP also risks barotrauma and detrimental effects on healthy and mildly injured alveoli [11,12]. Such damage negates positive effects and further complicates the patient condition and care. Thus, determining and setting optimal patient-specific PEEP during MV must balance risk and reward $[1,2]$.

One of the patient-specific PEEP selection method is through setting PEEP between lower inflection point (LIP) and upper inflection point (UIP) of the static compliance curve [13]. However, the patient-specific static compliance curve is highly variable with the LIP or UIP not identifiable during tidal ventilation. Thus, the application of this method remains limited because it requires additional protocol and interrupting care. Another patient-specific PEEP selection method was through monitoring respiratory mechanics during PEEP changes. In particular, it was found in studies that patientspecific PEEP set to minimal elastance (or maximum compliance) can improve oxygenation and balance lung recruitment and avoid overdistension [2,14-18].

This manuscript presents a software program, Clinical Utilisation of Respiratory Elastance Software (CURE Soft), equipped with a respiratory mechanics model to calculate patient-specific respiratory mechanics (respiratory elastance and resistance) from realtime data provided by the ventilator. CURE Soft is also embedded with a decision support system to titrate a patient-specific ventilator PEEP level at the bedside, using the calculated patient-specific respiratory mechanics. This software is developed in JAVA, and can be used in real-time connected to a Puritan Bennett 840 ventilator, or similar device. It also has an offline setting that allows re-simulation of the clinical situation for training purposes. CURE Soft is tested in a clinical settings and the findings are presented here for evaluation.

\section{Implementation}

The aim of CURE Soft is to provide clinicians with real-time information about a patient's respiratory mechanics based on current ventilator settings and breath-to-breath data in a simple graphical format. The information is translated into time-series and pressure 


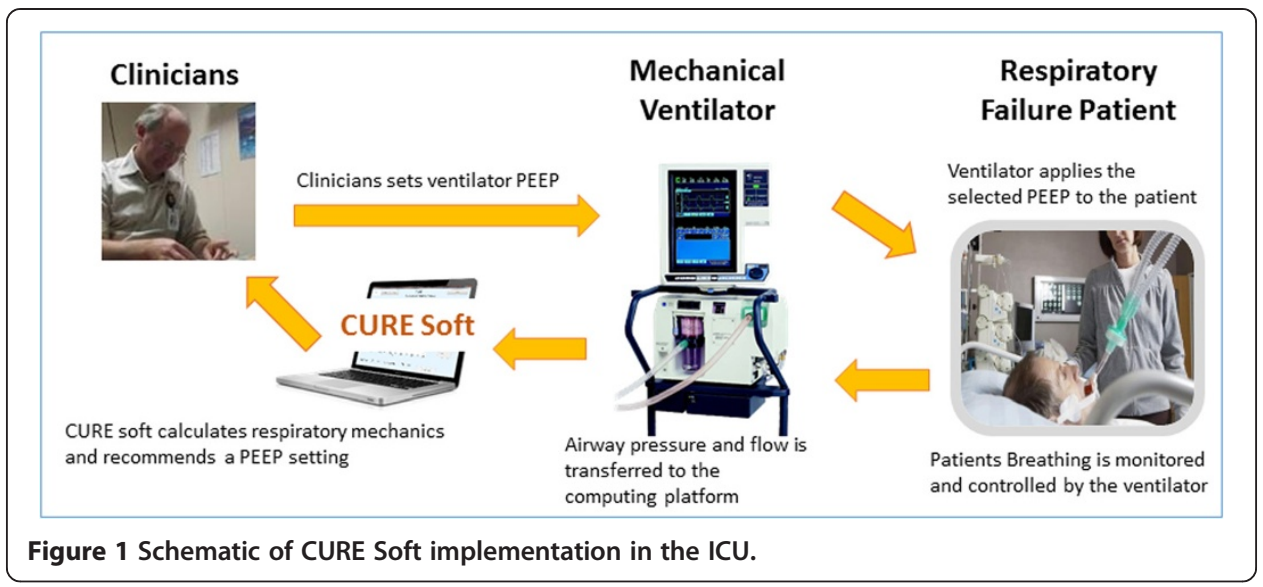

dependent graphs to help clinicians to select optimal ventilator settings. CURE Soft focuses on using model-identified, patient-specific respiratory mechanics parameters to help select a PEEP at minimal respiratory elastance $[2,18]$. Figure 1 shows a schematic of how CURE Soft is implemented in the intensive care unit (ICU) to support clinical decision making.

Time-varying elastance single compartment model

CURE Soft calculates respiratory mechanics parameters using an extended single compartment lung model which captures time-varying respiratory elastance model $[2,19]$. The model is described by Equations (1) and (2). This model is extended from the single compartment model and uses the measured airway pressure and flow of the ventilator to calculate the respiratory elastance and resistance for each breathing cycle. The model description can be found in Table 1.

$$
\begin{aligned}
& P_{a w}(t)=E_{r s} \times V(t)+R_{r s} \times Q(t)+P 0 \\
& E_{d r s}(t)=\left(P_{a w}(t)-P 0-R_{r s} \times Q(t)\right) / V(t)
\end{aligned}
$$

$V(t)$ is calculated by integrating the flow, $Q(t) . E_{r s}$ and $R_{r s}$ can be determined using multiple linear regression [19-20]. Using the $R_{r s}$ value obtained from Equation (1), the time-varying respiratory elastance $\left(E_{d r s}\right)$ within the inspiration of each breathing cycle is calculated using Equation (2) [19]. Each breathing cycle value for $E_{d r s}$ is then normalised to the total inspiration time, and the area under the $E_{d r s}$ curve ( $\left.A U C E_{d r s}\right)$ is used to calculate Stiffness (The term Stiffness is used in the CURE Soft for simpler understanding of non-engineering audience). This normalisation allows comparison across

Table 1 Model descriptions

\begin{tabular}{lll}
\hline Symbols & Description & Units \\
\hline$P_{a w}$ & Airway pressure in a breathing cycle & $\mathrm{cmH}_{2} \mathrm{O}$ \\
$t$ & Time & $\mathrm{s}$ \\
$E_{r s}$ & Respiratory system elastance & $\mathrm{CmH}_{2} \mathrm{O} / \mathrm{l}$ \\
$V$ & Air volume & $\mathrm{I}$ \\
$R_{r s}$ & Respiratory airway resistance & $\mathrm{cmH}_{2} \mathrm{Os} / \mathrm{l}$ \\
$Q$ & Airway flow & $\mathrm{I} / \mathrm{s}$ \\
$P O$ & Offset pressure & $\mathrm{CmH}_{2} \mathrm{O}$ \\
\hline
\end{tabular}


different respiratory rates within a given ventilation mode, or across different ventilation modes where respiratory rate may vary.

\section{Data acquisition, management and processing}

Airway pressure and flow data is obtained from the Puritan Bennett 840 ventilator through the ventilator's waveforms output function. The RS-232 serial port on the rear of the ventilator display is connected to a laptop running CURE Soft through an external USB adapter and a USB cable. The serial port was set up in JAVA with a baud rate of 38400, to match the ventilator output. A callback function is used to read all the available data and stores it in text format. In this data, two characters "BS" and "BE" can be found. These characters signify when breathing starts and ends respectively. "BS" appears when the ventilator starts to provide breathing support and "BE" appears at the end of the breathing cycle. Following the "BE", the starting point of a subsequent breath "BS", appears right after the previous "BE", and this cycles continue until the ventilator is disconnected or stopped providing support.

Patient-specific $E_{r s}$ is calculated from the single compartment lung model using only the inspiratory portion of breathing $[2,19]$. Each breathing cycle is separated into inspiration and expiratory sections. The occurrence of the "BS" character signifies the beginning of inspiration, and the next occurrence of these characters is the end of expiration. The end of inspiration is determined by searching for the first point where air flow changes from being positive to negative. In addition, breathing cycle filtering is performed, by quarantining 'abnormal' breathing cycles. These breathing cycles are too short, too long, non-physiological plausible or the breathing cycle data that are corrupted during data acquisition process. In particular, the filtering criteria are as follows:

- A stream of data that does not contain both $\mathrm{BS}$ and $\mathrm{BE}$

- A sudden pressure difference with more than $\pm 10 \mathrm{cmH}_{2} \mathrm{O}$ compared to previous point

- A sudden flow difference with more than $\pm 10 \mathrm{cmH}_{2} \mathrm{O} / \mathrm{l}$ compared to previous point

- A 'breathing cycle' with less than 5 data points is not processed

\section{Software architecture}

The architecture of the CURE Soft is designed according to the classical three-layer structure. The lowest layer is responsible for the persistent data storage and for the management of input ports. The input port management is isolated from the other parts of the system by dedicated objects in order to provide the opportunity of easy replacement of the used ventilator device. The middle layer implements the data processing and management functions in a modular way. Thus, the current set of functions and model calculations can be easily extended. The highest layer of the system provides the graphical user interface (GUI).

\section{Graphic user interface and main user function}

CURE Soft can be used in two modes of operation:

1) Online: This mode acquires data from the ventilator in real-time and calculates the respiratory mechanic parameters in real-time to aid clinical decision making. This mode also stores the real-time data and relevant input to a text format. 
2) Offline: This mode allows access to the data stored during online use for simulation. It provides the opportunity for user education purposes and auditing or reviewing patient care and progression.

The general procedure to execute CURE Soft is shown in Table 2.

Upon execution, the CURE Soft graphical user interface (GUI) is as shown in Figure 2. The CURE Soft GUI is separated into three sections: 1) Main Control, 2) Function and 3) Display. The buttons in Main Control are used for initiation and termination of the CURE Soft.

The Functions Panel is where the different CURE Soft functions are located. There are 6 main function tabs in the CURE Soft GUI with their functions summarised below:

1. History: Display the history of calculated Stiffness, PEEP and average Stiffness.

2. Recruitment: This tab is used during a recruitment manoeuvre (RM) or PEEP titration procedure. Stiffness at each PEEP is calculated and evaluated. The PEEP before minimal Stiffness or Stiffness occurs is selected as model-selected PEEP.

3. Events: This tab allows additional clinical events and procedures to be recorded.

4. Log: Log file for software evaluation purposes.

5. Settings: Settings for CURE Soft application. Settings include aspects like: 1) Online or Offline mode; 2) Where to store specific data files.

6. Individual Breath Cycle View: Allows every calculated parameter to be viewed at every evaluated breathing cycle.

Finally, the display section is used to present the results calculated from the models and data. A step-by-step guide in video format is included in the electronic supplemental file to provide offline training for the use of the CURE Soft application (See Additional files 1, 2, 3, 4, 5, 6 and 7).

\section{Software testing}

Unit and integration tests of CURE Soft were regularly executed during the development of the application. Occasionally, previously captured data sets were used for these

Table 2 CURE Soft application procedure

\begin{tabular}{|c|c|c|}
\hline Online mode & & Offline mode \\
\hline \multicolumn{3}{|l|}{ Ventilator settings } \\
\hline $\begin{array}{l}\text { 1. Check ventilator output, network communication, output waveform is } \\
\text { selected with baud rate } 38400 \text {. }\end{array}$ & & - \\
\hline $\begin{array}{l}\text { 2. Connect an RS232 serial port from the rear of the ventilator to the } \\
\text { computing platform with CURE Soft. }\end{array}$ & & - \\
\hline \multicolumn{3}{|l|}{ Computer settings } \\
\hline 1. Execute CURE Jar. & 1. & Execute CURE jar. \\
\hline 2. In settings tab, select serial port & 2. & $\begin{array}{l}\text { In settings tab, select } \\
\text { captured file. }\end{array}$ \\
\hline 3. Select the serial port connected to the RS232. & 3. & $\begin{array}{l}\text { Select the designated } \\
\text { captured file (*.txt). }\end{array}$ \\
\hline 4. Click 'start collecting data'. & 4. & Click 'start collecting data'. \\
\hline
\end{tabular}




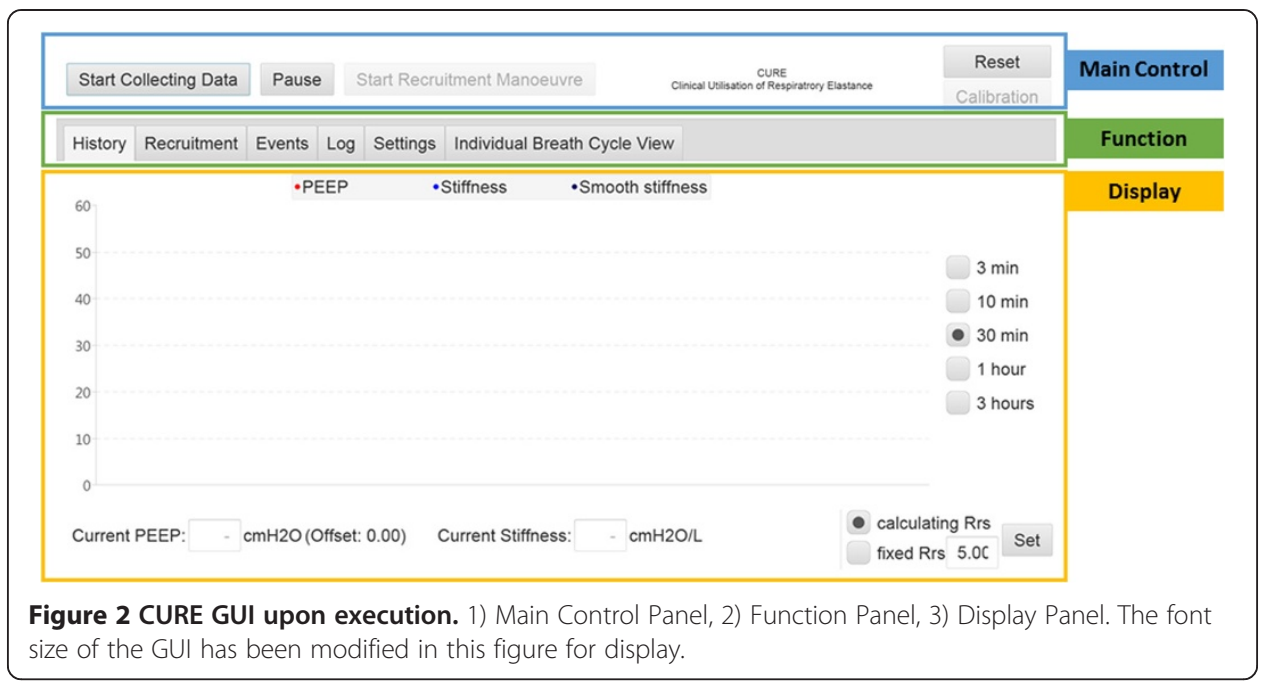

test cases. At the end of the development cycle, the functions of CURE Soft application were validated in two phases. First, CURE Soft was tested using a mechanical lung (Michigan Instruments Dual Adult Test Lung) connected the Puritan Bennett 840 (PB840) ventilator. To validate the CURE Soft application in clinical settings, a clinical trial is being carried out to investigate the potential of using respiratory mechanics to optimise PEEP in MV in Christchurch Hospital, New Zealand (Australian New Zealand Trial Registry Number: ACTRN12613001006730). Ethics approval for this study was granted by the New Zealand Southern Regional Ethics Committee (Reference number: 13/STH/84). Written informed consent was obtained from the patient for the publication of this report.

In this manuscript, the data from a patient who was mechanically ventilated invasively due to respiratory failure is included for software validation testing. This patient was intubated and ventilated using a Puritan Bennett 840 ventilator, under synchronous intermittent mandatory ventilation (SIMV) mode, volume control with tidal volume $(6 \sim 8 \mathrm{ml} / \mathrm{kg})$. The patient was later sedated and paralysed to undergo a RM for PEEP titration. These sections of data are used to demonstrate the program and its functionality.

It is important to note that while a PB840 ventilator is used in the development and testing, the software is fully general. Using the architecture as described above, implementation of the CURE Soft in other ventilators only requires changing the data acquisition and processing to allows access to pressure and flow data of that specific ventilator.

\section{Results and discussion}

\section{CURE Soft trial and general observations}

At the start of the clinical trial, CURE Soft is initiated to calculate respiratory system stiffness. Figure 3 shows the respiratory mechanics calculated for the patient over different periods of time. The blue line shows the calculated respiratory system $A U C E_{d r s}$, or 'stiffness'. The black line indicates is the smooth stiffness averaged over the last 60 breathing cycles. The red line is the PEEP detected from the ventilator airway pressure. The result shown in Figure 3 is divided into 4 sections. 


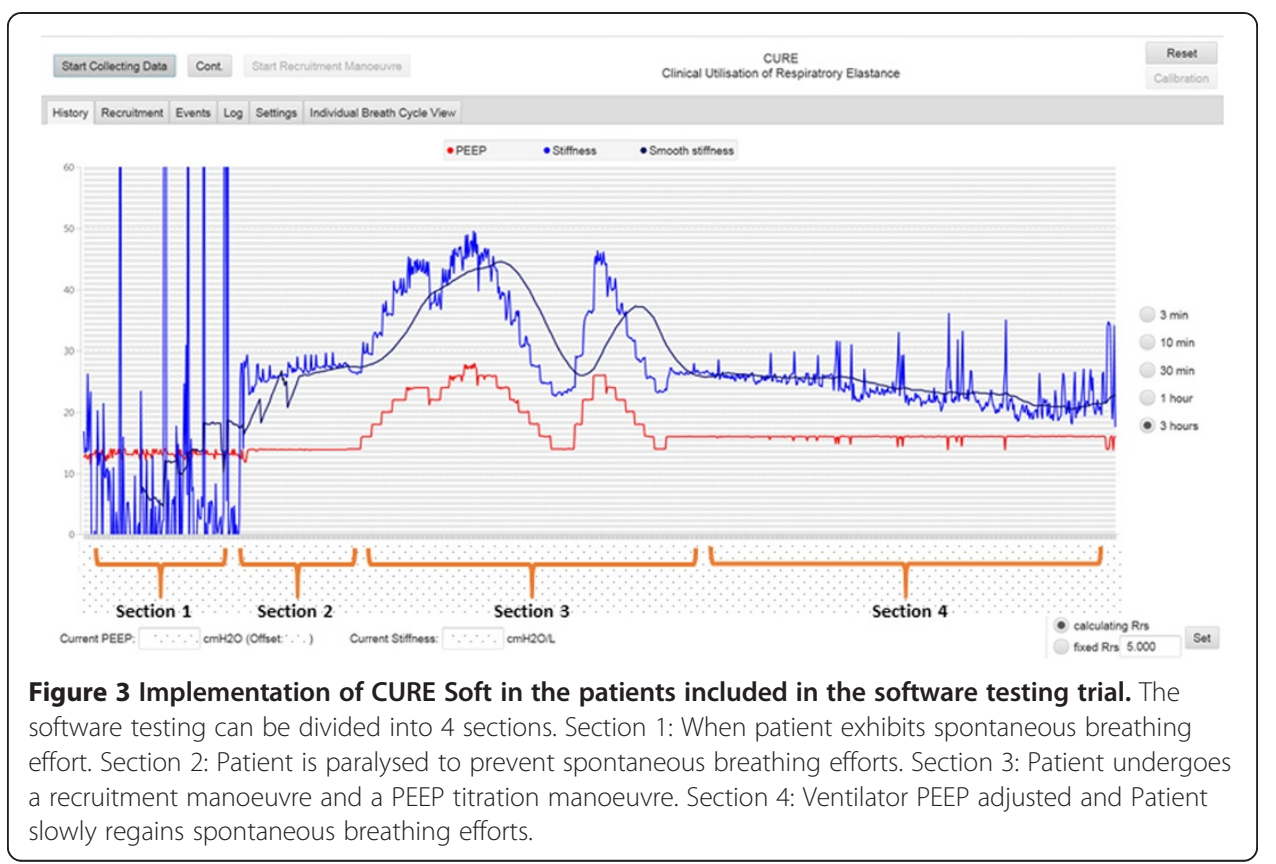

- Section 1: Respiratory mechanics were calculated at the start of the clinical trial and patient was spontaneously breathing on top of ventilator support.

- Section 2: Patient is paralysed to prevent spontaneous breathing efforts.

- Section 3: Once patient is paralysed, a protocolised RM is performed. The PEEP is increased in steps of $2 \mathrm{cmH}_{2} \mathrm{O}$ until peak airway pressure reached $55 \mathrm{cmH}_{2} \mathrm{O}$, then decrease back to initial PEEP. A second RM is performed to titrate PEEP and validate the initial findings during the first RM.

- Section 4: Ventilator PEEP is selected based on the CURE Soft recommendation and clinician consensus. Patient slowly regain spontaneous breathing efforts.

In Section 1, the patient respiratory stiffness fluctuates and is sometimes reduced to non-physiological value of less than zero. These non-physiological values occurred due to the patient's added spontaneous breathing effort [21,22]. Patients who are spontaneously breathing are more variable, and thus, each breathing cycle is altered by the variable pressure generated by the diaphragm and/or intercostal muscles. These variable efforts significantly altered the airway pressure and flow data, affecting the respiratory mechanics and parameter identification process. The ability of CURE Soft to calculate an effective, net patient-specific respiratory mechanics values is limited when the patient is breathing spontaneously due to incorrect modelling approach [23]. When the patient is spontaneously breathing, there is a need of additional measuring tools to isolate patient-specific breathing effort for patient-specific respiratory mechanics calculation [24].

In Section 2, the patient was given muscle relaxants for paralysis. Once the patient was paralysed, the stiffness fluctuation in Section 1 ceased almost immediately. From this point, the true patient-specific respiratory mechanics are no longer altered by the patient's inspiratory effort, and the mechanical parameter values are reliable.

Figure 4 shows the airway pressure, flow, volume and calculated time-varying elastance $\left(E_{d r s}\right)$ curve for several breathing cycles before and after paralysis. It is important 


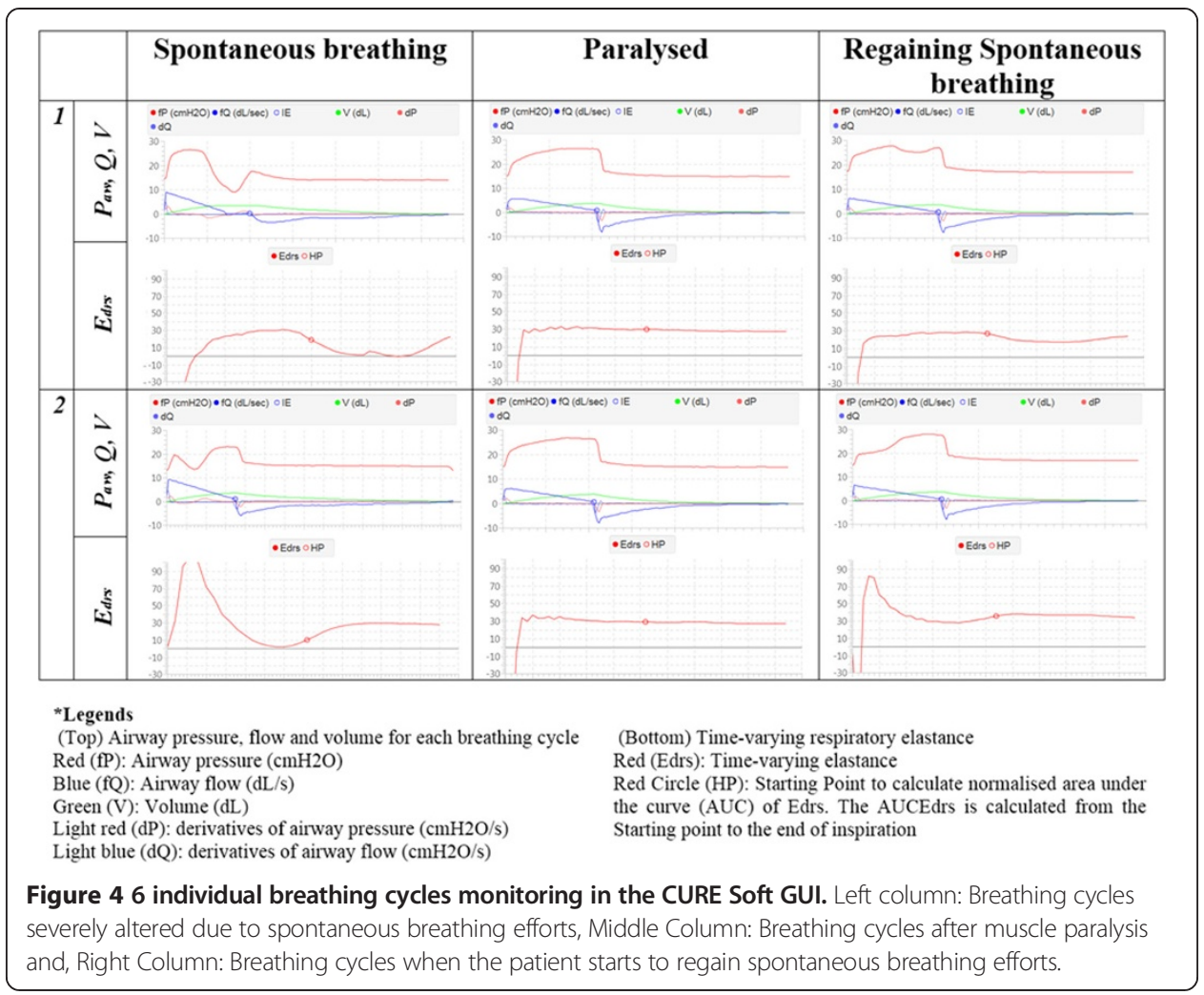

to note that, if the calculated respiratory mechanics are used to guide ventilation, the respiratory mechanics model must provide unique solutions without a wide range of variation. Thus, in this study, since the model was not able to account for patient variability during Section 1 of the trial, patients was given muscle relaxants to reduce this variability.

Once the patient was paralysed and the respiratory mechanics could reliably be calculated, a step-wise PEEP increase RM was performed to evaluate the respiratory stiffness at each PEEP level $[2,19]$. A second RM was also performed for validation and PEEP titration. At the start of the first RM, the 'Start Recruitment Manoeuvre' button is clicked, and the CURE Soft user is required to calibrate the ventilator displayed PEEP with actual measured PEEP. After the PEEP is calibrated, an event log is also available to record the patient's position, protocol, pulse oximetry oxygen saturation $\left(\mathrm{SpO}_{2}\right)$ and fraction of inspired oxygen $\left(\mathrm{FiO}_{2}\right)$. The calibration dialog and event logs are shown in Figure 5.

After the events are recorded, the History Tab display is switched to the Recruitment Tab display. This tab displays the respiratory system stiffness on the y-axis and with PEEP on $x$-axis as shown in Figures 6 and 7. The plots in Figures 6 and 7 correspond to the respiratory mechanics in Section 3, Figure 3.

During the first RM as shown in Figure 6, the PEEP was increased step-wise from initial value of $14 \mathrm{cmH}_{2} \mathrm{O}$ until PEEP level of $27.5 \mathrm{cmH}_{2} \mathrm{O}$. The PEEP is then decreased step-wise to the initial PEEP setting. The purpose of the RM is to recruit collapsed lung. As the PEEP is decreased back to the initial PEEP, there is a noticeable hysteresis between stiffness during PEEP increase, and stiffness during PEEP decrease. This hysteresis indicates additional alveoli recruitment. As PEEP is decreased from higher PEEP, 


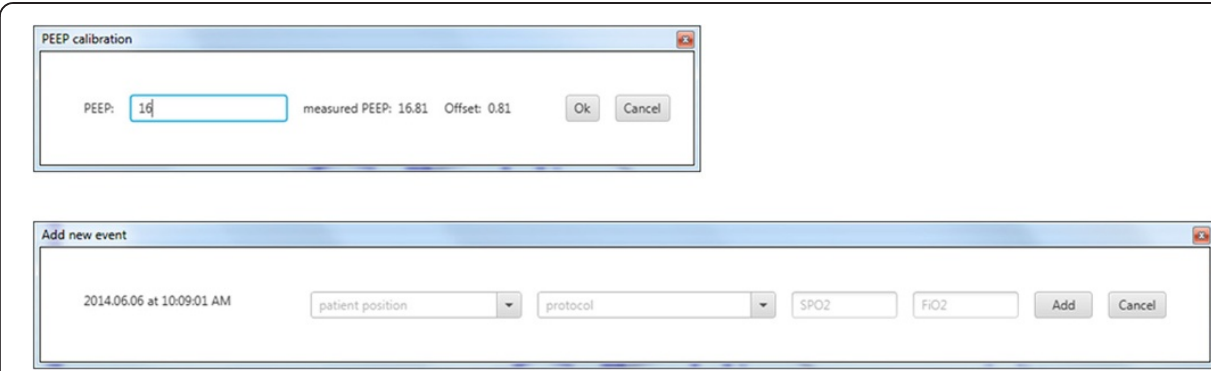

Figure 5 CURE GUI pop-up dialog. Top: PEEP calibration and, Bottom: Events Log.

the threshold closing pressures of these newly recruited alveoli are lower than their threshold opening pressure, and thus these alveoli remain opened.

At the end of the RM, the 'Stop Recruitment Manoeuvre' button in Figure 6 is clicked. This action will trigger the PEEP selection function, where a PEEP will be suggested by the CURE Soft based on inflection elastance (in this case, a PEEP of 15 $\mathrm{cmH}_{2} \mathrm{O}$ is recommended) [2]. This CURE Soft suggested PEEP occurs right before the minimal elastance (stiffness) PEEP, which aim to maintain time-dependent alveoli recruitment and a balance of recruitment and overdistension [19]. The user is then given an option to accept the suggested PEEP or not, as shown in Figure 8. CURE Soft GUI aims to provide decision support in PEEP selection. It is important that the final clinical decision is confirmed in CURE Soft by the attending clinicians. Following the first RM, a second RM was performed to titrate PEEP again using the 'Start Recruitment Manoeuvre' button. The result of the second RM is as shown in Figure 7. The first or previous RM stiffness-PEEP plot is recorded in the background of the display for comparison with the newer RM. During the second RM and compared to the previous RM, it is obvious that the stiffness-PEEP curve during increasing and decreasing PEEP were effectively overlaid. This result suggested that the first RM was able to 'fully' recruit the

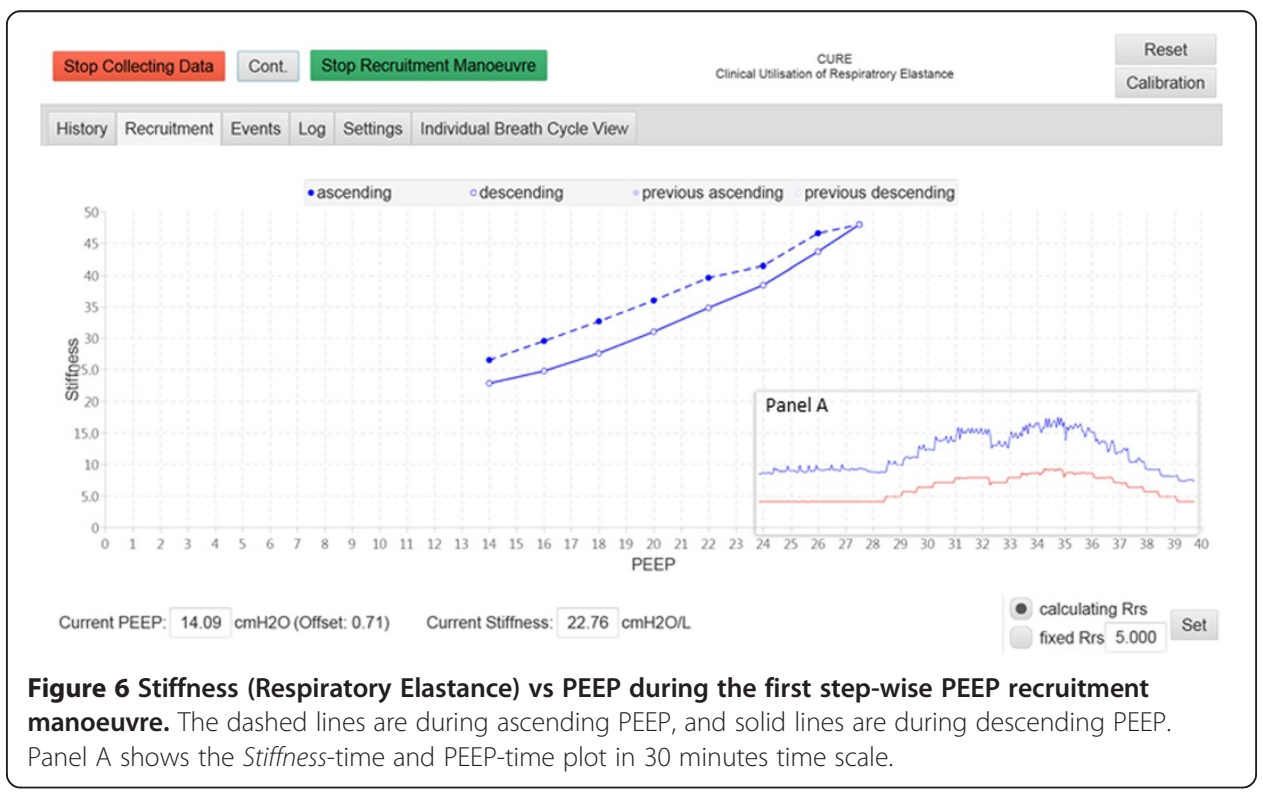




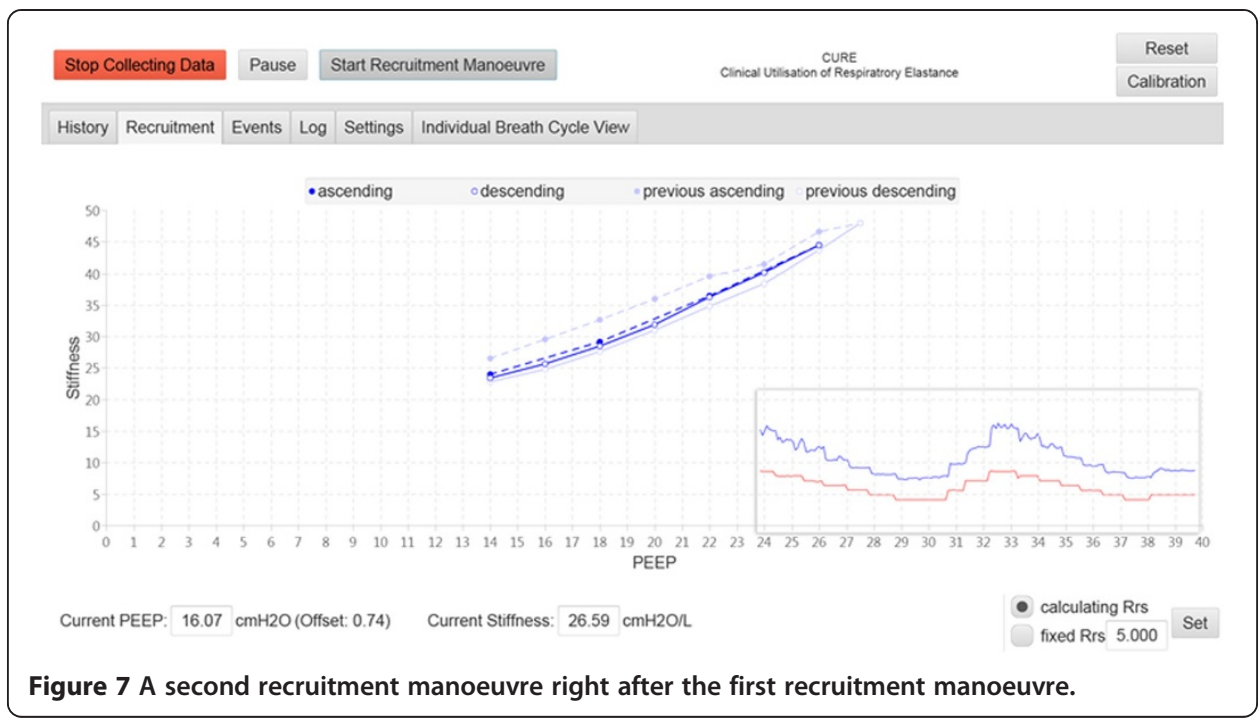

recruitable collapsed alveoli and there is relatively little or no alveoli collapse after the first RM. At the end of the RM, the Stiffness-PEEP curve is again evaluated and a PEEP of $15 \mathrm{cmH}_{2} \mathrm{O}$ is recommended as optimal ventilator PEEP. However, as this was a trial for software testing, and attending clinicians decided to select a PEEP of $16 \mathrm{cmH}_{2} \mathrm{O}$.

After the PEEP was set at $16 \mathrm{cmH}_{2} \mathrm{O}$, the RMs were stopped, but the patient's respiratory mechanics, together with airway pressure and flow profiles, were continuously recorded until the end of Section 4 as shown in Figure 3. From Section 3 to Section 4, it was found that the respiratory stiffness fluctuates at the end of the trial. This result suggested that the patient started to regain spontaneous breathing effort. This fluctuation of respiratory stiffness corresponds to the 'entrainment' of breathing on MV as shown in Figure 4 (Right Column) where the shape of the airway pressure is altered by the patients breathing effort [21,22].

\section{Application of CURE Soft in respiratory mechanics monitoring}

CURE Soft identifies patient-specific respiratory mechanics (elastance or stiffness) in real-time from ventilator data. Calculations of respiratory mechanics were previously available by performing specific intensive clinical protocols [25]. The existing methods of respiratory mechanics monitoring are invasive and/or interrupt care, and thus cannot be performed continuously. In particular, most ventilator continuous monitoring methods use two point respiratory mechanics estimation, which can only be performed in one specific set ventilation mode using an additional end of inspiratory pause (EIP) [26], which can be automated by some ventilators (For example: the Engstrom Carestation and Puritan Bennett 980). Equally, this method heavily relies on the duration of the EIP $[26,27]$ and provides only a local estimation at that point and pressure of the breath, which may not match what is obtained via a model-based approach that requires no EIP. Thus, in comparison, CURE Soft uses a model-based approach using readily available airway pressure and flow profile, adds no additional burden to patients, and provides more information than was previously unavailable. Clinically, the CURE Soft system allows continuous tracking of patients' respiratory mechanics across time. 


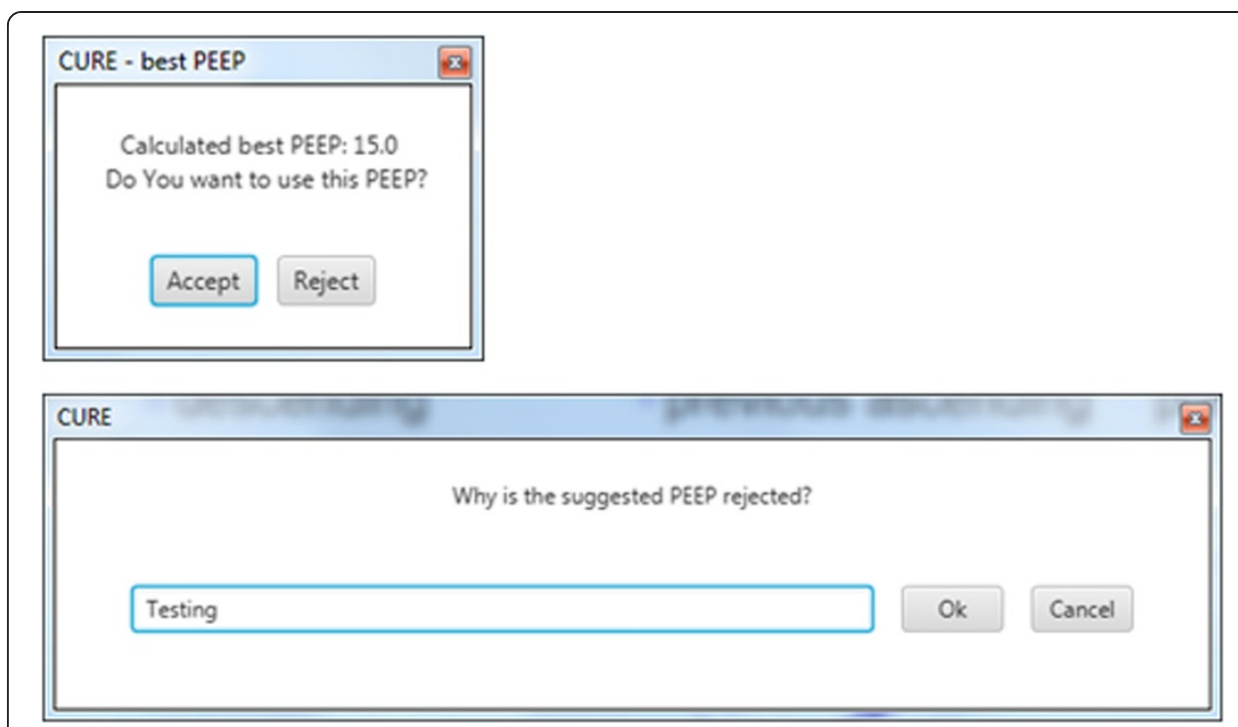

Figure 8 PEEP is recommended to the CURE Soft user once 'Stop Recruitment Manoeuvre' button is clicked. It is at the user discretion to follow the suggestion.

Information about changing patient condition, and response to treatment over time is very useful, as it can be used to evaluate the efficacy of previous treatments and regularly audit patient care and progression.

CURE Soft also provides real-time feedback on the effect of a RM, and allows clinical staff to determine a patients' lung recruitability, and suggest an optimal patient- and time- specific PEEP level. Finally, as shown here, monitoring respiratory mechanics continuously can also provide information about patient-ventilator interactions and thus, potentially provide useful insight to anaesthesia, analgesia and overall patient ventilator management that was not necessarily previously available to clinicians.

\section{Limitations and future works}

CURE Soft was able to calculate patient-specific respiratory mechanics for every breath using the airway pressure and flow information from the ventilator. However, as shown in the results, the single compartment model used by CURE Soft is less reliable if the patient is breathing spontaneously, although this issue can be managed [28]. In particular, patients with spontaneous breathing, create a pressure or flow drop during a breathing cycle, as shown in Figure 4 (Left and Right Column). This pressure drop alters the shape of the airway pressure or flow curve of that breathing cycle, and thus, the calculated respiratory mechanics are over or under estimated. While this limitation can be overcome with the use of muscle relaxants, it was reported that the use of muscle relaxants are generally not clinically feasible and a more advanced models are required [28]. Thus, the application of CURE Soft is currently limited to patients who are synchronised with the ventilation support without 'entrainment' [22]. However, the addition of the advanced model presents no hurdle or significant change to the existing GUI.

In this study, the CURE Soft was tested in human trials using a SIMV volume control mode with ramp flow profile. This flow profile is similar to the flow profile generated during pressure control mode. Thus, the CURE Soft has similar ability to capture the 
respiratory mechanics during pressure control modes. This ability was tested under different controlled ventilation using the mechanical lung (see Additional file 1). However, the application of CURE Soft during pressure control mode warrants further investigation in human subjects.

There is also a need to further study of the effect of optimising PEEP on patient recovery. CURE Soft selects a PEEP slightly higher than the minimal elastance PEEP as seen in Figure 7. This higher PEEP is selected to maintain alveoli recruitment aside from maintaining a balance in recruitment and overdistension [19]. A recent study by Pintado et al. found that selecting PEEP based on a metric similar to minimal respiratory elastance may potentially improve patient recovery [29]. Thus, the application of CURE Soft, is a system necessary to test this hypothesis at scale without clinically intensive and thus, infrequent interventions.

\section{Conclusions}

CURE Soft enables the real-time calculation of model-based respiratory mechanics for patients receiving mechanical ventilation. It is also capable of providing a unique training and auditing tool for clinical users. Initial results from the clinical trials, showed that the system is able to provide detailed, previously unavailable information on patient-specific respiratory mechanics and response to therapy in real-time. The additional insight available to clinicians provides the potential for improved decisionmaking, and thus improved patient care and outcomes.

\section{Availability and requirements}

Project name: Clinical Utilisation of Respiratory Elastance Software (CURE Soft)

Project home page: not available

Operating system(s): Windows, Linux

Programming language: JAVA 7

Other requirements: JAVA JRE7, Mechanical Ventilator (CURE Soft was tested on Puritan Bennett 840 and Puritan Bennett 980)

Any restrictions to use by non-academics: no restrictions

\section{Additional files}

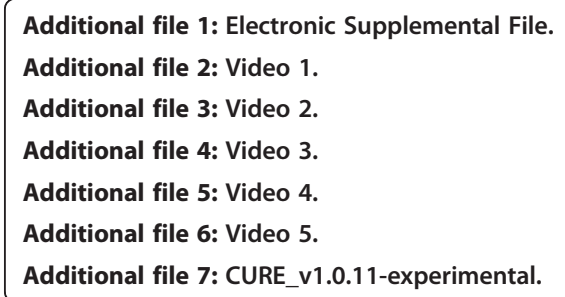

\section{Abbreviations}

ARDS: Acute respiratory distress syndrome; CURE: Clinical utilisation of respiratory elastance; USB: Universal serial bus; Soft: Software; GUl: Graphic user interface; $E_{d r s}$ : Time-varying respiratory elastance; $E_{r s}$ : Respiratory system elastance; $R_{r s}$ : Respiratory system resistance; $P_{a w}$ : Airway pressure; $Q$ : Airway flow; V: Air volume; PO: Offset pressure or positive end-expiratory pressure; PB840: Puritan Bennett 840 ventilator; MV: Mechanical ventilation; $\mathrm{SpO}_{2}$ : Pulse oximetry oxygen saturation; $\mathrm{FiO}_{2}$ : Fraction of inspired oxygen; PEEP: Positive end-expiratory pressure; $\mathrm{RM}$ : Recruitment manoeuvre; ICU: Intensive care unit. 


\section{Authors' contributions}

AS designed and developed the software and help draft the manuscript. YSC tested the software and help draft the manuscript. DR, AB, DG, SC and VM collected the data and tested the software. CP, BB, TD and JGC had inputs to the software design and helped draft the manuscript. All authors read and approved the final manuscript.

\section{Authors' information}

AS and BB works at Department of Control Engineering and Information, Budapest University of Technology and Economics. YSC, DR, AB, DG, SC, VM, CP and JGC are from the Centre for BioEngineering, University of Canterbury. GMS works in the Department of Intensive care in Christchurch Hospital. TD works at the GIGA-Cardiovascular Sciences, University of Liege.

\section{Acknowledgements}

The authors wish to thank the Health Research Council of New Zealand for supporting this research. The authors would also like to thank members of the Centre for BioEngineering for their aid in testing and feedback of the software.

\section{Author details}

Department of Control Engineering and Information, Budapest University of Technology and Economics, Budapest, Hungary. ${ }^{2}$ Centre for BioEngineering, University of Canterbury, Canterbury, New Zealand. ${ }^{3}$ Department of Intensive Care, Christchurch Hospital, Canterbury, New Zealand. ${ }^{4}$ GIGA Cardiovascular Science, University of Liege, Liege, Belgium.

Received: 9 July 2014 Accepted: 20 September 2014

Published: 30 September 2014

\section{References}

1. Sundaresan A, Chase JG: Positive end expiratory pressure in patients with acute respiratory distress syndrome - The past, present and future. Biomed Signal Process Control 2011, 7(2):93-103.

2. Chiew YS, Chase JG, Shaw G, Sundaresan A, Desaive T: Model-based PEEP optimisation in mechanical ventilation. BioMed Eng OnLine 2011, 10(1):111.

3. Kallet RH, Branson RD: Do the NIH ARDS clinical trials network PEEP/FIO2 tables provide the best evidence-based guide to balancing PEEP and FIO2 settings in adults? Respir Care 2007, 52(4):461-477.

4. Chase JG, Moeller K, Shaw G, Schranz C, Chiew YS, Desaive T: When the value of gold is zero. BMC Res Notes 2014, 7(1):404.

5. The Acute Respiratory Distress Syndrome Network: Ventilation with lower tidal volumes as compared with traditional tidal volumes for acute lung injury and the acute respiratory distress syndrome. N Engl J Med 2000 342(18):1301-1308.

6. Mercat A, Richard J-CM, Vielle B, Jaber S, Osman D, Diehl J-L, Lefrant J-Y, Prat G, Richecoeur J, Nieszkowska A, Gervais C, Baudot J, Bouadma L, Brochard L, Group EPES: Positive End-expiratory pressure setting in adults with acute lung injury and acute respiratory distress syndrome: a randomized controlled trial. JAMA 2008, 299(6):646-655.

7. Meade MO, Cook DJ, Guyatt GH, Slutsky AS, Arabi YM, Cooper DJ, Davies AR, Hand LE, Zhou Q, Thabane L, Austin P, Lapinsky S, Baxter A, Russell J, Skrobik Y, Ronco JJ, Stewart TE, for the Lung Open Ventilation Study I: Ventilation strategy using Low tidal volumes, recruitment maneuvers, and high positive End-expiratory pressure for acute lung injury and acute respiratory distress syndrome: a randomized controlled trial. JAMA 2008, 299(6):637-645.

8. Brower RG, Lanken PN, Maclntyre N, Matthay MA, Morris A, Ancukiewicz M, Schoenfeld D, Thompson BT: Higher versus lower positive End-expiratory pressures in patients with the acute respiratory distress syndrome. $N$ Engl J Med 2004, 351(4):327-336.

9. Briel M, Meade M, Mercat A, Brower RG, Talmor D, Walter SD, Slutsky AS, Pullenayegum E, Zhou Q, Cook D, Brochard L, Richard J-CM, Lamontagne F, Bhatnagar N, Stewart TE, Guyatt G: Higher vs lower positive end-expiratory pressure in patients with acute lung injury and acute respiratory distress syndrome: Systematic review and meta-analysis. JAMA 2010, 303(9):865-873.

10. Gattinoni L, Carlesso E, Brazzi L, Caironi P: Positive end-expiratory pressure. Curr Opin Crit Care 2010, 16(1):39-44.

11. Slutsky AS: Lung injury caused by mechanical ventilation*. Chest 1999, 116(suppl 1):95-15S.

12. Lucangelo U, Pelosi P, Zin W, Aliverti A, Crimi E, Sorbo L, Ranieri VM: Ventilator-Associated Lung Injury. In Respiratory System and Artificial Ventilation. Milan: Springer; 2008:119-137.

13. Albaiceta G, Garcia E, Taboada F: Comparative study of four sigmoid models of pressure-volume curve in acute lung injury. BioMed Eng OnLine 2007, 6(1):7.

14. Mols G, Brandes I, Kessler V, Lichtwarck-Aschoff M, Loop T, Geiger K, Guttmann J: Volume-dependent compliance in ARDS: proposal of a new diagnostic concept. Intensive Care Med 1999, 25:1084-1091.

15. Suarez-Sipmann F, Bohm SH, Tusman G, Pesch T, Thamm O, Reissmann H, Reske A, Magnusson A, Hedenstierna G Use of dynamic compliance for open lung positive end-expiratory pressure titration in an experimental study. Crit Care Med 2007, 35:214-221.

16. Carvalho A, Jandre F, Pino A, Bozza F, Salluh J, Rodrigues R, Ascoli F, Giannella-Neto A: Positive end-expiratory pressure at minimal respiratory elastance represents the best compromise between mechanical stress and lung aeration in oleic acid induced lung injury. Crit Care 2007, 11(4):R86.

17. Schumann S, Vimlati L, Kawati R, Guttmann J, Lichtwarck Aschoff M: Analysis of dynamic intratidal compliance in a lung collapse model. Anesthesiology 2011, 114(5):1111-1117. doi:1110.1097/ALN.1110b1013e31820ad31841b.

18. Lambermont B, Ghuysen A, Janssen N, Morimont P. Hartstein G, Gerard P, D'Orio V: Comparison of functional residual capacity and static compliance of the respiratory system during a positive end-expiratory pressure 
(PEEP) ramp procedure in an experimental model of acute respiratory distress syndrome. Crit Care 2008, 12(4):R91.

19. van Drunen E, Chiew YS, Pretty C, Shaw G, Lambermont B, Janssen N, Chase JG, Desaive T: Visualisation of time-varying respiratory system elastance in experimental ARDS animal models. BMC Pulm Med 2014, 14(1):33.

20. Lucangelo U, Bernabè F, Blanch L: Lung mechanics at the bedside: make it simple. Curr Opin Crit Care 2007, 13(1):64-72.

21. Gilstrap D, Maclntyre N: Patient-ventilator interactions. Implications for clinical management. Am J Respir Crit Care Med 2013, 188(9):1058-1068.

22. Akoumianaki E, Lyazidi A, Rey N, Matamis D, Perez-Martinez N, Giraud R, Mancebo J, Brochard L, Richard J-CM: Mechanical ventilation-induced reverse-triggered breaths: A frequently unrecognized form of neuromechanical coupling. CHEST 2013, 143(4):927-938.

23. Brochard L, Martin G, Blanch L, Pelosi P, Belda FJ, Jubran A, Gattinoni L, Mancebo J, Ranieri VM, Richard J-C, Gommers D, Vieillard-Baron A, Pesenti A, Jaber S, Stenqvist O, Vincent J-L: Clinical review: Respiratory monitoring in the ICU - a consensus of 16. Crit Care 2012, 16(2):219.

24. Khirani S, Polese G, Aliverti A, Appendini L, Nucci G, Pedotti A, Colledan M, Lucianetti A, Baconnier P, Rossi A: On-line monitoring of lung mechanics during spontaneous breathing: a physiological study. Respir Med 2010, 104(3):463-471.

25. Albaiceta GM, Piacentini E, Villagr $\tilde{A}_{i}$ A, Lopez-Aguilar J, Taboada F, Blanch L: Application of continuous positive airway pressure to trace static pressure-volume curves of the respiratory system. Crit Care Med 2003, 31(10):2514-2519.

26. van Drunen E, Chiew YS, Chase JG, Shaw G, Lambermont B, Janssen N, Damanhuri N, Desaive T: Expiratory model-based method to monitor ARDS disease state. BioMed Eng OnLine 2013, 12(1):57.

27. Barberis L, Manno E, Guérin C: Effect of end-inspiratory pause duration on plateau pressure in mechanically ventilated patients. Intensive Care Med 2003, 29(1):130-134.

28. Chiew YS: Model-Based Mechanical Ventilation for the Critically III. New Zealand: University of Canterbury; 2013.

29. Pintado M-C, de Pablo R, Trascasa M, Milicua J-M, Rogero S, Daguerre M, Cambronero J-A, Arribas I, Sánchez-García M: Individualized PEEP setting in subjects with ARDS: a randomized controlled pilot study. Respir Care 2013, 58(9):1416-1423.

doi:10.1186/1475-925X-13-140

Cite this article as: Szlavecz et al.: The Clinical Utilisation of Respiratory Elastance Software (CURE Soft): a bedside software for real-time respiratory mechanics monitoring and mechanical ventilation management. BioMedical Engineering OnLine 2014 13:140.

\section{Submit your next manuscript to BioMed Central and take full advantage of:}

- Convenient online submission

- Thorough peer review

- No space constraints or color figure charges

- Immediate publication on acceptance

- Inclusion in PubMed, CAS, Scopus and Google Scholar

- Research which is freely available for redistribution

Submit your manuscript at www.biomedcentral.com/submit 